IJMMS 30:9 (2002) 569-574

PII. S016117120200786X

http://ijmms.hindawi.com

(c) Hindawi Publishing Corp.

\title{
CERTAIN INTEGRAL OPERATOR AND STRONGLY STARLIKE FUNCTIONS
}

\author{
JIN-LIN LIU
}

Received 15 June 2001

Let $S^{*}(\rho, \gamma)$ denote the class of strongly starlike functions of order $\rho$ and type $\gamma$ and let $C(\rho, \gamma)$ be the class of strongly convex functions of order $\rho$ and type $\gamma$. By making use of an integral operator defined by Jung et al. (1993), we introduce two novel families of strongly starlike functions $S_{\beta}^{\alpha}(\rho, \gamma)$ and $C_{\beta}^{\alpha}(\rho, \gamma)$. Some properties of these classes are discussed.

2000 Mathematics Subject Classification: 30C45, 30C75.

1. Introduction. Let $A$ denote the class of functions of the form

$$
f(z)=z+\sum_{n=2}^{\infty} a_{n} z^{n}
$$

which are analytic in the unit disc $E=\{z:|z|<1\}$. A function $f(z)$ belonging to $A$ is said to be starlike of order $\gamma$ if it satisfies

$$
\operatorname{Re}\left\{\frac{z f^{\prime}(z)}{f(z)}\right\}>\gamma \quad(z \in E)
$$

for some $\gamma(0 \leq \gamma<1)$. We denote by $S^{*}(\gamma)$ the subclass of $A$ consisting of functions which are starlike of order $\gamma$ in $E$. Also, a function $f(z)$ in $A$ is said to be convex of order $\gamma$ if it satisfies $z f^{\prime}(z) \in S^{*}(\gamma)$, or

$$
\operatorname{Re}\left\{1+\frac{z f^{\prime \prime}(z)}{f^{\prime}(z)}\right\}>\gamma \quad(z \in E)
$$

for some $\gamma(0 \leq \gamma<1)$. We denote by $C(\gamma)$ the subclass of $A$ consisting of all functions which are convex of order $\gamma$ in $E$.

If $f(z) \in A$ satisfies

$$
\left|\arg \left(\frac{z f^{\prime}(z)}{f(z)}-\gamma\right)\right|<\frac{\pi}{2} \rho \quad(z \in E)
$$

for some $\gamma(0 \leq \gamma<1)$ and $\rho(0<\rho \leq 1)$, then $f(z)$ is said to be strongly starlike of order $\rho$ and type $\gamma$ in $E$, and denoted by $f(z) \in S^{*}(\rho, \gamma)$. If $f(z) \in A$ satisfies

$$
\left|\arg \left(1+\frac{z f^{\prime \prime}(z)}{f^{\prime}(z)}-\gamma\right)\right|<\frac{\pi}{2} \rho \quad(z \in E)
$$


for some $\gamma(0 \leq \gamma<1)$ and $\rho(0<\rho \leq 1)$, then we say that $f(z)$ is strongly convex of order $\rho$ and type $\gamma$ in $E$, and we denote by $C(\rho, \gamma)$ the class of such functions. It is clear that $f(z) \in A$ belongs to $C(\rho, \gamma)$ if and only if $z f^{\prime}(z) \in S^{*}(\rho, \gamma)$. Also, we note that $S^{*}(1, \gamma)=S^{*}(\gamma)$ and $C(1, \gamma)=C(\gamma)$.

For $c>-1$ and $f(z) \in A$, we recall the generalized Bernardi-Libera-Livingston integral operator $L_{c}(f)$ as

$$
L_{c}(f)=\frac{c+1}{z^{c}} \int_{0}^{z} t^{c-1} f(t) d t
$$

The operator $L_{c}(f)$ when $c \in N=\{1,2,3, \ldots\}$ was studied by Bernardi [1]. For $c=1$, $L_{1}(f)$ was investigated by Libera [4].

Recently, Jung et al. [2] introduced the following one-parameter family of integral operators:

$$
Q_{\beta}^{\alpha} f(z)=\left(\begin{array}{c}
\alpha+\beta \\
\beta
\end{array}\right) \frac{\alpha}{z^{\beta}} \int_{0}^{z}\left(1-\frac{t}{z}\right)^{\alpha-1} t^{\beta-1} f(t) d t \quad(\alpha>0, \beta>-1, f \in A) .
$$

They showed that

$$
Q_{\beta}^{\alpha} f(z)=z+\sum_{n=2}^{\infty} \frac{\Gamma(\beta+n) \Gamma(\alpha+\beta+1)}{\Gamma(\beta+\alpha+n) \Gamma(\beta+1)} a_{n} z^{n}
$$

where $\Gamma(x)$ is the familiar Gamma function. Some properties of this operator have been studied (see [2, 3]). From (1.7) and (1.8), one can see that

$$
z\left(Q_{\beta}^{\alpha+1} f(z)\right)^{\prime}=(\alpha+\beta+1) Q_{\beta}^{\alpha} f(z)-(\alpha+\beta) Q_{\beta}^{\alpha+1} f(z)
$$

It should be remarked in passing that the operator $Q_{\beta}^{\alpha}$ is related rather closely to the Beta or Euler transformation.

Using the operator $Q_{\beta}^{\alpha}$, we now introduce the following classes:

$$
\begin{aligned}
& S_{\beta}^{\alpha}(\rho, \gamma)=\left\{f(z) \in A: Q_{\beta}^{\alpha} f(z) \in S^{*}(\rho, \gamma), \frac{z\left(Q_{\beta}^{\alpha} f(z)\right)^{\prime}}{Q_{\beta}^{\alpha} f(z)} \neq \gamma \forall z \in E\right\}, \\
& C_{\beta}^{\alpha}(\rho, \gamma)=\left\{f(z) \in A: Q_{\beta}^{\alpha} f(z) \in C(\rho, \gamma), \frac{\left(z\left(Q_{\beta}^{\alpha} f(z)\right)^{\prime}\right)^{\prime}}{\left(Q_{\beta}^{\alpha} f(z)\right)^{\prime}} \neq \gamma \forall z \in E\right\} .
\end{aligned}
$$

It is obvious that $f(z) \in C_{\beta}^{\alpha}(\rho, \gamma)$ if and only if $z f^{\prime}(z) \in S_{\beta}^{\alpha}(\rho, \gamma)$.

In this note, we investigate some properties of the classes $S_{\beta}^{\alpha}(\rho, \gamma)$ and $C_{\beta}^{\alpha}(\rho, \gamma)$. The basic tool for our investigation is the following lemma which is due to Nunokawa [5].

LEMMA 1.1. Let a function $p(z)=1+c_{1} z+c_{2} z^{2}+\cdots$ be analytic in $E$ and $p(z) \neq 0$ $(z \in E)$. If there exists a point $z_{0} \in E$ such that

$$
|\arg p(z)|<\frac{\pi}{2} \rho \quad\left(|z|<\left|z_{0}\right|\right), \quad\left|\arg p\left(z_{0}\right)\right|=\frac{\pi}{2} \rho \quad(0<\rho \leq 1),
$$


then

$$
\frac{z_{0} p^{\prime}\left(z_{0}\right)}{p\left(z_{0}\right)}=i k \rho
$$

where

$$
\begin{aligned}
& k \geq \frac{1}{2}\left(a+\frac{1}{a}\right) \quad\left(\text { when } \arg p\left(z_{0}\right)=\frac{\pi}{2} \rho\right), \\
& k \leq-\frac{1}{2}\left(a+\frac{1}{a}\right) \quad\left(\text { when } \arg p\left(z_{0}\right)=-\frac{\pi}{2} \rho\right),
\end{aligned}
$$

and $p\left(z_{0}\right)^{1 / \rho}= \pm i a(a>0)$.

2. Main results. Our first inclusion theorem is stated as follows.

THEOREM 2.1. The class $S_{\beta}^{\alpha}(\rho, \gamma) \subset S_{\beta}^{\alpha+1}(\rho, \gamma)$ for $\alpha>0, \beta>-1,0 \leq \gamma<1$ and $\alpha+\beta \geq-\gamma$.

Proof. Let $f(z) \in S_{\beta}^{\alpha}(\rho, \gamma)$. Then we set

$$
\frac{z\left(Q_{\beta}^{\alpha+1} f(z)\right)^{\prime}}{Q_{\beta}^{\alpha+1} f(z)}=(1-\gamma) p(z)+\gamma
$$

where $p(z)=1+c_{1} z+c_{2} z^{2}+\cdots$ is analytic in $E$ and $p(z) \neq 0$ for all $z \in E$. Using (1.9) and (2.1), we have

$$
(\alpha+\beta+1) \frac{Q_{\beta}^{\alpha} f(z)}{Q_{\beta}^{\alpha+1} f(z)}=(\alpha+\beta+\gamma)+(1-\gamma) p(z) .
$$

Differentiating both sides of (2.2) logarithmically, it follows from (2.1) that

$$
\frac{z\left(Q_{\beta}^{\alpha} f(z)\right)^{\prime}}{Q_{\beta}^{\alpha} f(z)}-\gamma=(1-\gamma) p(z)+\frac{(1-\gamma) z p^{\prime}(z)}{(\alpha+\beta+\gamma)+(1-\gamma) p(z)} .
$$

Suppose that there exists a point $z_{0} \in E$ such that

$$
|\arg p(z)|<\frac{\pi}{2} \rho \quad\left(|z|<\left|z_{0}\right|\right), \quad\left|\arg p\left(z_{0}\right)\right|=\frac{\pi}{2} \rho .
$$

Then, by applying Lemma 1.1 , we can write that $z_{0} p^{\prime}\left(z_{0}\right) / p\left(z_{0}\right)=i k \rho$ and that $\left(p\left(z_{0}\right)\right)^{1 / \rho}= \pm i a(a>0)$.

Therefore, if $\arg p\left(z_{0}\right)=-(\pi / 2) \rho$, then

$$
\begin{aligned}
\frac{z_{0}\left(Q_{\beta}^{\alpha} f\left(z_{0}\right)\right)^{\prime}}{Q_{\beta}^{\alpha} f\left(z_{0}\right)}-\gamma & =(1-\gamma) p\left(z_{0}\right)\left[1+\frac{z_{0} p^{\prime}\left(z_{0}\right) / p\left(z_{0}\right)}{(\alpha+\beta+\gamma)+(1-\gamma) p\left(z_{0}\right)}\right] \\
& =(1-\gamma) a^{\rho} e^{-i \pi \rho / 2}\left[1+\frac{i k \rho}{(\alpha+\beta+\gamma)+(1-\gamma) a^{\rho} e^{-i \pi \rho / 2}}\right]
\end{aligned}
$$


From (2.5) we have

$$
\begin{aligned}
& \arg \left\{\frac{z_{0}\left(Q_{\beta}^{\alpha} f\left(z_{0}\right)\right)^{\prime}}{Q_{\beta}^{\alpha} f\left(z_{0}\right)}-\gamma\right\} \\
& =-\frac{\pi}{2} \rho+\arg \left\{1+\frac{i k \rho}{\left.(\alpha+\beta+\gamma)+(1-\gamma) a^{\rho} e^{-i \pi \rho / 2}\right\}}\right. \\
& =-\frac{\pi}{2} \rho+\tan ^{-1}\left\{\left(k \rho\left[(\alpha+\beta+\gamma)+(1-\gamma) a^{\rho} \cos \frac{\pi \rho}{2}\right]\right)\right. \\
& \quad \times\left((\alpha+\beta+\gamma)^{2}+2(\alpha+\beta+\gamma)(1-\gamma) a^{\rho} \cos \frac{\pi \rho}{2}\right. \\
& \left.\left.\quad+(1-\gamma)^{2} a^{2 \rho}-k \rho(1-\gamma) a^{\rho} \sin \frac{\pi \rho}{2}\right)^{-1}\right\} \\
& \leq-\frac{\pi}{2} \rho,
\end{aligned}
$$

where $k \leq-(1 / 2)(a+1 / a) \leq-1, \alpha+\beta \geq-\gamma$, which contradicts the condition $f(z) \in$ $S_{\beta}^{\alpha}(\rho, \gamma)$.

Similarly, if $\arg p\left(z_{0}\right)=(\pi / 2) \rho$, then we have

$$
\arg \left\{\frac{z_{0}\left(Q_{\beta}^{\alpha} f\left(z_{0}\right)\right)^{\prime}}{Q_{\beta}^{\alpha} f\left(z_{0}\right)}-\gamma\right\} \geq \frac{\pi}{2} \rho,
$$

which also contradicts the hypothesis that $f(z) \in S_{\beta}^{\alpha}(\rho, \gamma)$.

Thus the function $p(z)$ has to satisfy $|\arg p(z)|<(\pi / 2) \rho(z \in E)$, which leads us to the following:

$$
\left|\arg \left\{\frac{z\left(Q_{\beta}^{\alpha+1} f(z)\right)^{\prime}}{Q_{\beta}^{\alpha+1} f(z)}-\gamma\right\}\right|<\frac{\pi}{2} \rho \quad(z \in E) .
$$

This evidently completes the proof of Theorem 2.1.

We next state the following theorem.

THEOREM 2.2. The class $C_{\beta}^{\alpha}(\rho, \gamma) \subset C_{\beta}^{\alpha+1}(\rho, \gamma)$ for $\alpha>0, \beta>-1,0 \leq \gamma<1$, and $\alpha+\beta \geq-\gamma$.

Proof. By definition (1.10), we have

$$
\begin{aligned}
f(z) \in C_{\beta}^{\alpha}(\rho, \gamma) & \Longleftrightarrow Q_{\beta}^{\alpha} f(z) \in C(\rho, \gamma) \Longleftrightarrow z\left(Q_{\beta}^{\alpha} f(z)\right)^{\prime} \in S^{*}(\rho, \gamma) \\
& \Longleftrightarrow Q_{\beta}^{\alpha}\left(z f^{\prime}(z)\right) \in S^{*}(\rho, \gamma) \Longleftrightarrow z f^{\prime}(z) \in S_{\beta}^{\alpha}(\rho, \gamma) \\
& \Longleftrightarrow z f^{\prime}(z) \in S_{\beta}^{\alpha+1}(\rho, \gamma) \Longleftrightarrow Q_{\beta}^{\alpha+1}\left(z f^{\prime}(z)\right) \in S^{*}(\rho, \gamma) \\
& \Longleftrightarrow z\left(Q_{\beta}^{\alpha+1} f(z)\right)^{\prime} \in S^{*}(\rho, \gamma) \Longleftrightarrow Q_{\beta}^{\alpha+1} f(z) \in C(\rho, \gamma) \\
& \Longleftrightarrow f(z) \in C_{\beta}^{\alpha+1}(\rho, \gamma) .
\end{aligned}
$$

The following theorem involves the generalized Bernardi-Libera-Livingston integral operator $L_{c}(f)$ given by (1.6). 
THEOREM 2.3. Let $c>-\gamma$ and $0 \leq \gamma<1$. If $f(z) \in A$ and $z\left(Q_{\beta}^{\alpha} L_{c} f(z)\right)^{\prime} / Q_{\beta}^{\alpha} L_{c} f(z)$ $\neq \gamma$ for all $z \in E$, then $f(z) \in S_{\beta}^{\alpha}(\rho, \gamma)$ implies that $L_{c}(f) \in S_{\beta}^{\alpha}(\rho, \gamma)$.

Proof. Let $f(z) \in S_{\beta}^{\alpha}(\rho, \gamma)$. Put

$$
\frac{z\left(Q_{\beta}^{\alpha} L_{c} f(z)\right)^{\prime}}{Q_{\beta}^{\alpha} L_{c} f(z)}=\gamma+(1-\gamma) p(z),
$$

where $p(z)$ is analytic in $E, p(0)=1$ and $p(z) \neq 0(z \in E)$. From (1.6) we have

$$
z\left(Q_{\beta}^{\alpha} L_{c} f(z)\right)^{\prime}=(c+1) Q_{\beta}^{\alpha} f(z)-c Q_{\beta}^{\alpha} L_{c} f(z) .
$$

Using (2.10) and (2.11), we get

$$
(c+1) \frac{Q_{\beta}^{\alpha} f(z)}{Q_{\beta}^{\alpha} L_{c} f(z)}=(c+\gamma)+(1-\gamma) p(z) .
$$

Differentiating both sides of (2.12) logarithmically, we obtain

$$
\frac{z\left(Q_{\beta}^{\alpha} f(z)\right)^{\prime}}{Q_{\beta}^{\alpha} f(z)}-\gamma=(1-\gamma) p(z)+\frac{(1-\gamma) z p^{\prime}(z)}{(c+\gamma)+(1-\gamma) p(z)} .
$$

Suppose that there exists a point $z_{0} \in E$ such that

$$
|\arg p(z)|<\frac{\pi}{2} \rho \quad\left(|z|<\left|z_{0}\right|\right), \quad\left|\arg p\left(z_{0}\right)\right|=\frac{\pi}{2} \rho .
$$

Then, applying Lemma 1.1, we can write that $z_{0} p^{\prime}\left(z_{0}\right) / p\left(z_{0}\right)=i k \rho$ and $\left(p\left(z_{0}\right)\right)^{1 / \rho}=$ $\pm i a(a>0)$.

If $\arg p\left(z_{0}\right)=(\pi / 2) \rho$, then

$$
\begin{aligned}
\frac{z_{0}\left(Q_{\beta}^{\alpha} f\left(z_{0}\right)\right)^{\prime}}{Q_{\beta}^{\alpha} f\left(z_{0}\right)}-\gamma & =(1-\gamma) p\left(z_{0}\right)\left[1+\frac{z_{0} p^{\prime}\left(z_{0}\right) / p\left(z_{0}\right)}{(c+\gamma)+(1-\gamma) p\left(z_{0}\right)}\right] \\
& =(1-\gamma) a^{\rho} e^{i \pi \rho / 2}\left[1+\frac{i k \rho}{(c+\gamma)+(1-\gamma) a^{\rho} e^{i \pi \rho / 2}}\right] .
\end{aligned}
$$

This shows that

$$
\begin{aligned}
\arg \left\{\frac{z_{0}\left(Q_{\beta}^{\alpha} f\left(z_{0}\right)\right)^{\prime}}{Q_{\beta}^{\alpha} f\left(z_{0}\right)}-\gamma\right\} & \\
= & \frac{\pi}{2} \rho+\arg \left\{1+\frac{i k \rho}{(c+\gamma)+(1-\gamma) a^{\rho} e^{i \pi \rho / 2}}\right\} \\
= & \frac{\pi}{2} \rho+\tan ^{-1}\left\{\left(k \rho\left[(c+\gamma)+(1-\gamma) a^{\rho} \cos \frac{\pi \rho}{2}\right]\right)\right. \\
& \times\left((c+\gamma)^{2}+2(c+\gamma)(1-\gamma) a^{\rho} \cos \frac{\pi \rho}{2}\right. \\
& \left.\left.+(1-\gamma)^{2} a^{2 \rho}+k \rho(1-\gamma) a^{\rho} \sin \frac{\pi \rho}{2}\right)^{-1}\right\} \\
\geq \frac{\pi}{2} \rho, &
\end{aligned}
$$

where $k \geq(1 / 2)(a+1 / a) \geq 1$, which contradicts the condition $f(z) \in S_{\beta}^{\alpha}(\rho, \gamma)$. 
Similarly, we can prove the case $\arg p\left(z_{0}\right)=-(\pi / 2) \rho$. Thus we conclude that the function $p(z)$ has to satisfy $|\arg p(z)|<(\pi / 2) \rho$ for all $z \in E$. This shows that

$$
\left|\arg \left\{\frac{z\left(Q_{\beta}^{\alpha} L_{c} f(z)\right)^{\prime}}{Q_{\beta}^{\alpha} L_{c} f(z)}-\gamma\right\}\right|<\frac{\pi}{2} \rho \quad(z \in E) .
$$

The proof is complete.

THEOREM 2.4. Let $c>-\gamma$ and $0 \leq \gamma<1$. If $f(z) \in A$ and $\left(z\left(Q_{\beta}^{\alpha} L_{c} f(z)\right)^{\prime}\right)^{\prime} /$ $\left(Q_{\beta}^{\alpha} L_{c} f(z)\right)^{\prime} \neq \gamma$ for all $z \in E$, then $f(z) \in C_{\beta}^{\alpha}(\rho, \gamma)$ implies that $L_{c}(f) \in C_{\beta}^{\alpha}(\rho, \gamma)$.

Proof. Using the same method as in Theorem 2.2 we have

$$
\begin{aligned}
f(z) \in C_{\beta}^{\alpha}(\rho, \gamma) & \Longleftrightarrow z f^{\prime}(z) \in S_{\beta}^{\alpha}(\rho, \gamma) \Longrightarrow L_{c}\left(z f^{\prime}(z)\right) \in S_{\beta}^{\alpha}(\rho, \gamma) \\
& \Longleftrightarrow z\left(L_{c} f(z)\right)^{\prime} \in S_{\beta}^{\alpha}(\rho, \gamma) \Longleftrightarrow L_{c} f(z) \in C_{\beta}^{\alpha}(\rho, \gamma) .
\end{aligned}
$$

\section{REFERENCES}

[1] S. D. Bernardi, Convex and starlike univalent functions, Trans. Amer. Math. Soc. 135 (1969), 429-446.

[2] I. B. Jung, Y. C. Kim, and H. M. Srivastava, The Hardy space of analytic functions associated with certain one-parameter families of integral operators, J. Math. Anal. Appl. 176 (1993), no. 1, 138-147.

[3] J. L. Li, Some properties of two integral operators, Soochow J. Math. 25 (1999), no. 1, 91-96.

[4] R. J. Libera, Some classes of regular univalent functions, Proc. Amer. Math. Soc. 16 (1965), 755-758.

[5] M. Nunokawa, On properties of non-Carathéodory functions, Proc. Japan Acad. Ser. A Math. Sci. 68 (1992), no. 6, 152-153.

Jin-Lin LiU: DePARTMENT OF MATHEMATICS, YANGZHOU UNIVERSiTY, YANGZHOU 225002, JIANGSU, CHINA 


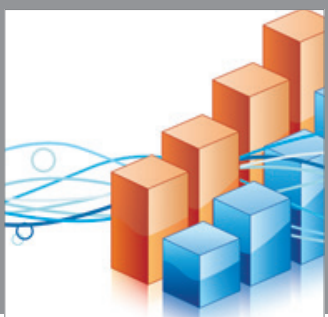

Advances in

Operations Research

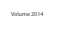

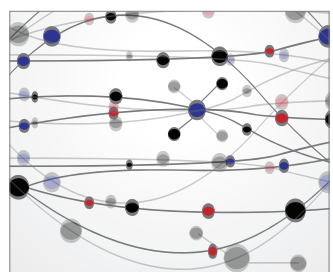

\section{The Scientific} World Journal
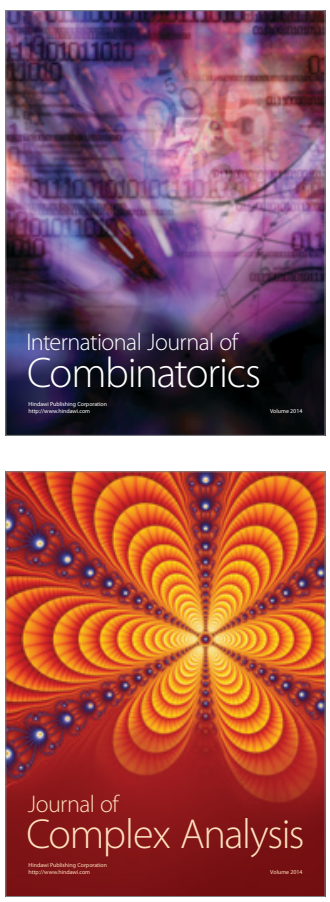

International Journal of

Mathematics and

Mathematical

Sciences
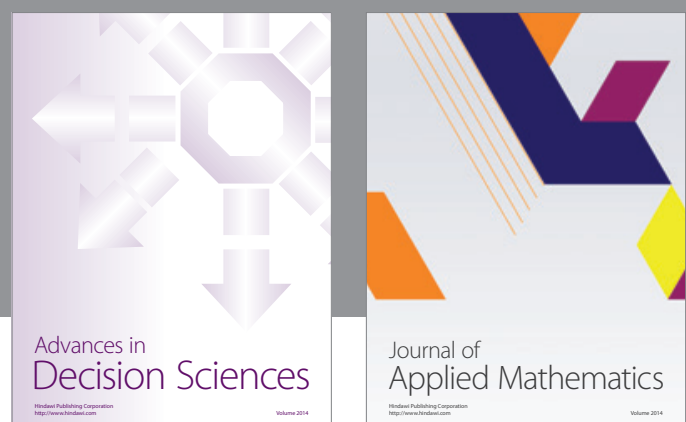

Journal of

Applied Mathematics
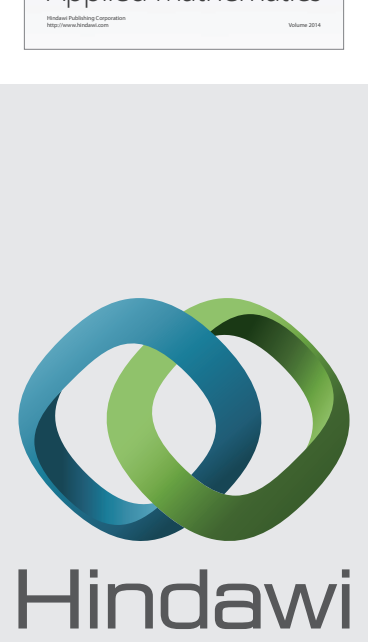

Submit your manuscripts at http://www.hindawi.com
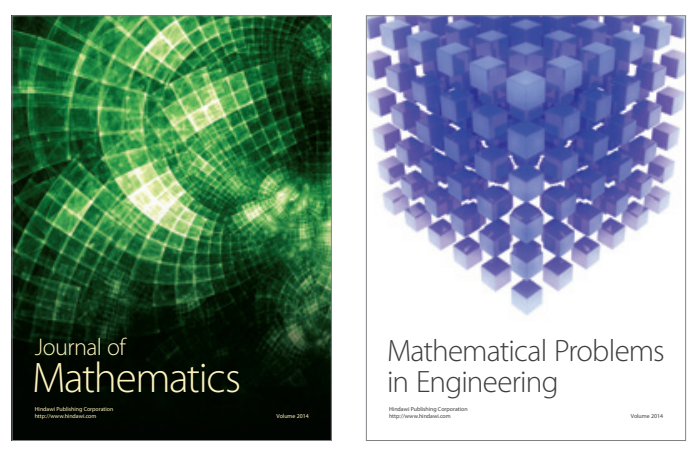

Mathematical Problems in Engineering
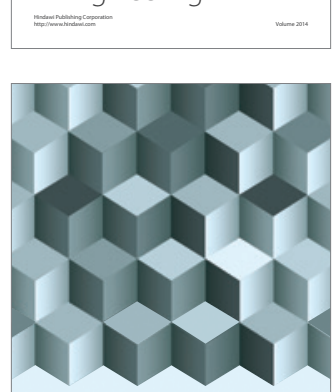

Journal of

Function Spaces
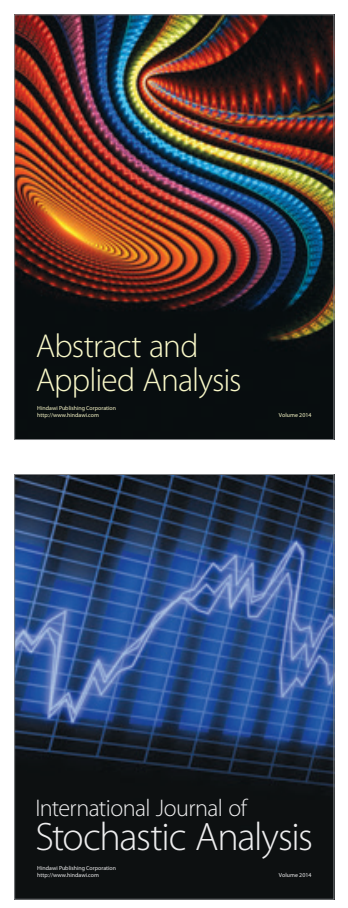

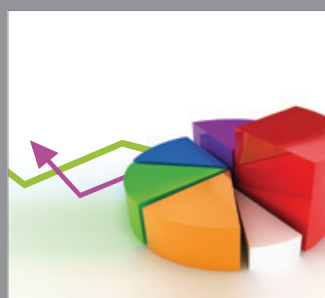

ournal of

Probability and Statistics

Promensencen
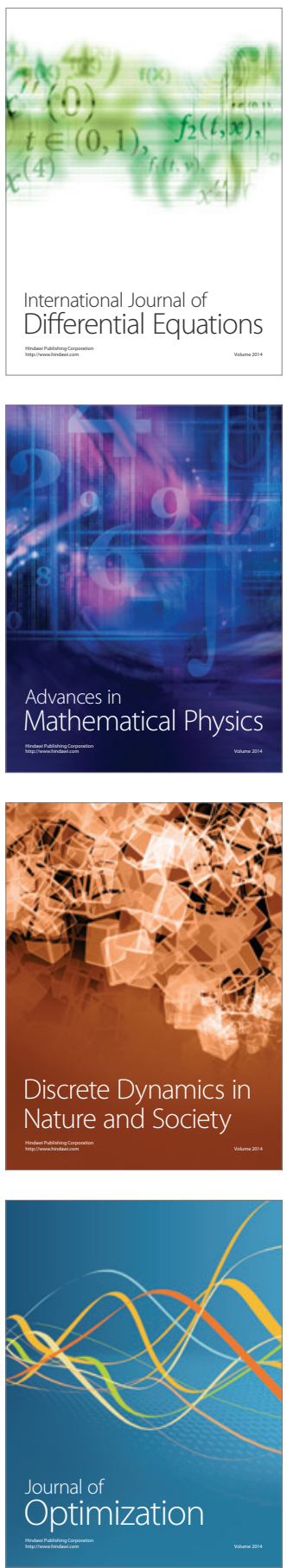